Thorax (1950), 5, 138.

\title{
LEIOMYOMA OF THE BRONCHUS
}

\author{
BY
}

\author{
S. I. TURKINGTON, G. A. SCOTT, AND T. B. SMILEY \\ From the Royal Victoria Hospital, Belfast
}

The bronchial tree is a most unusual site for a leiomyoma, and the rarity of such a tumour justifies the presentation of this case.

\section{CASE REPORT}

The patient was a housewife aged 57 years. In 1942 she suffered from severe metrorrhagia stated to be caused by uterine fibroids. A subtotal hysterectomy was performed in a country hospital, but the pathological details of this are not available. Since this operation and until the onset of the illness to be described, the patient was in excellent health.

In July 1949, whilst a front-seat passenger in one of two colliding motor-cars, the patient's left side came into violent contact with the dashboard. Sustaining apparently minor abrasions of the left arm and recovering from the shock, she went home feeling

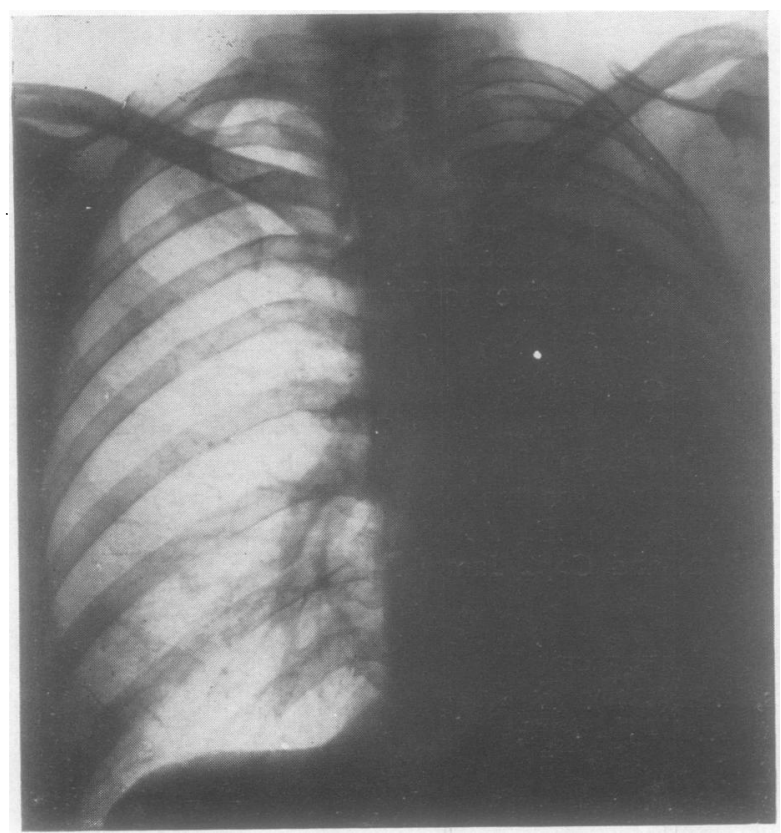

FIG. 1.-Postero-anterior view of the chest taken on admission showing massive collapse of the left lung, with mediastinal displacement. well. That evening she noticed gradually increasing breathlessness and, in the early hours of the following morning, marked dyspnoea which confined her to bed. A harsh spasmodic cough developed, with the expectoration of a scanty but " rusty" sputum. Symptomatic treatment during the ensuing two months gave her little or no relief.

In September, 1949, the patient was admitted to the Royal Victoria Hospital, Belfast.

Examination.-She was a wellnourished woman of medium build, restless and cyanosed, harassed by frequent spasms of unproductive coughing. There was no evidence of anaemia or clubbing of the fingers.

Clinical examination revealed the signs of collapse of the left lung, and this was confirmed radiologically (Fig. 1). 
A pelvi-rectal examination was carried out and the gynaecological operation mentioned above was thus confirmed, but no other abnormality was noted.

Differential Diagnosis.-The presence of a foreign body in the bronchus was not substantiated by the history. The patient had nothing in the mouth at the time of the accident except her dentures, and inspection revealed these to be intact. In view of the history, the symptoms, the clinical and radiological findings, fracture of the bronchus was thought to be a possibility. Finally bronchial neoplasm was considered, with the possibility of trauma contributing to the final occlusion of the bronchus by disturbing the growth in situ, or by causing it to bleed into the bronchial lumen.

Progress.- On the day following admission, a bronchoscopic examination was carried out (T. B. S.), and the findings were as follows. The cords, trachea, and carina were normal. There was no sub-carinal widening. The left main bronchus was angulated upwards, and there was a block a few centimetres below the carina caused by blood clot and debris. On clearing this, using suction and biopsy forceps, the left lower lobe bronchus was seen to be patent, but there was a raw necrotic surface on the posterior wall of this bronchus, just below the level of the left upper lobe orifice. A biopsy specimen was taken from this region, and this material proved to be old thrombus.

Following bronchoscopy there was symptomatic and clinical improvement with relief from the incessant cough. Air entry was now noted to be present in the upper lobe (Fig. 2). However the improvement was temporary. Within a few days the symptoms returned. A hectic intermittent temperature developed with a polymorphonuclear leucocytosis of 15,000 per c.mm. The physical signs were those of complete collapse of the left lung. A second bronchoscopy was therefore performed. The findings were similar to those described above, except that on this occasion when the thrombus and debris were removed, a quantity of pus escaped from the left lower bronchi. Again clinical and symptomatic improvement resulted, but this was not maintained.

The biopsy specimen taken at this examination showed squamous metaplasia and pleomorphic cells of a predominantly spindle celled type; a bronchial neoplasm was suspected.

The patient's condition deteriorated and pneumonectomy was performed (T. B. S.) on October $18,1949$.

Post-operative progress was uneventful.

Pathological Report.-Dr. J. Edgar Morison reported as follows:

The entire left lung has been received and fixed by injecting a solution containing $10 \%$

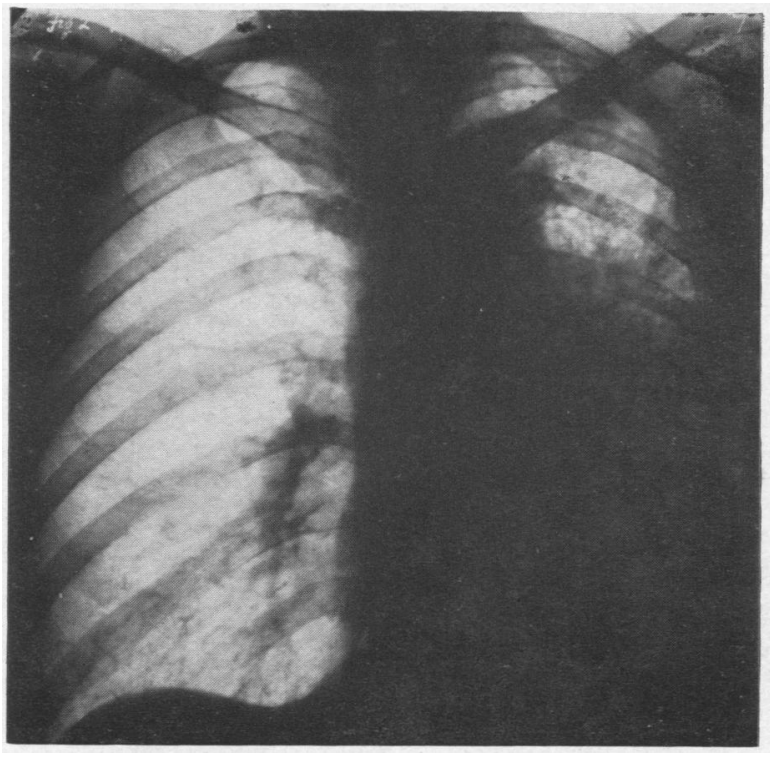

Fig. 2.-Radiograph after bronchoscopy showing aeration of the left upper lobe with some correction of the mediastinal displacement. 
formalin into the main bronchus and its divisions. On opening the lower lobe bronchus a polypoid mass was found projecting out of its apical division and growing towards the hilum, but this only slightly narrowed the main lower lobe bronchus at this point. This mass was covered with blood clot and its surface was ulcerated and irregular. It extended along the apical bronchus towards the periphery of the lung, filling the lumen of the bronchus and the majority of its primary and secondary divisions (Fig. 3). It dilated these bronchi and compressed their wall so that they resembled thin-walled blood vessels. The adjacent subapical bronchus was small and the mass in the apical bronchus had compressed it, and apparently obliterated its lumen. Mucoid secretions had accumulated in some of the divisions of this bronchus and in some of the more peripheral divisions of the apical bronchus beyond the

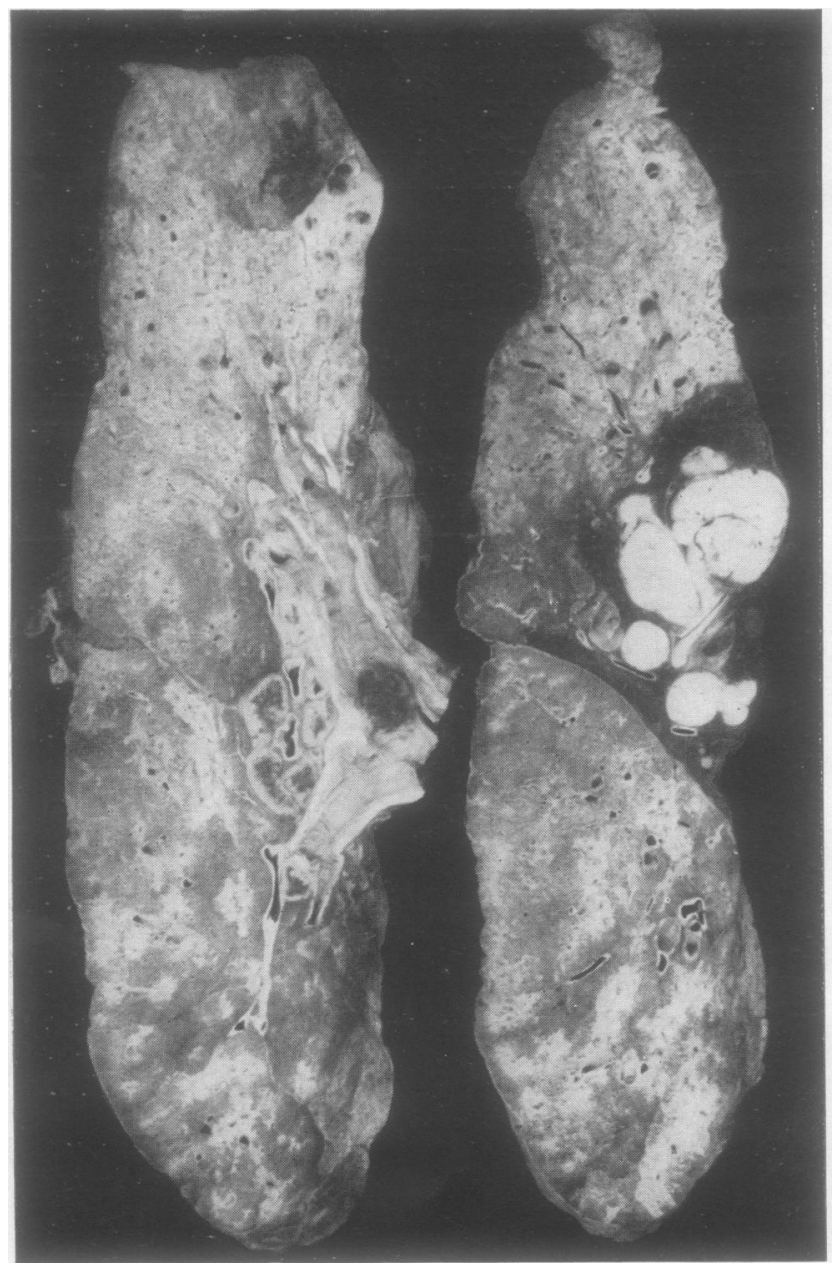

Fig. 3.-The section on the right shows the tumour projecting into the lower lobe bronchus, that on the left is more posterior and shows the tumour growing peripherally in the dilated bronchi. obstructing tumour. In areas, often remote from the tumour and in both upper and lower lobes, but more especially in the lower lobe, there were firm areas, the largest about $1 \mathrm{~cm}$. in diameter, but many about the size of a pin head. In these areas the lung structure was poorly seen or replaced by greyish white tissue. The large arteries and veins of the lung were normal. The hilar lymph glands were not significantly enlarged.

Histological examination showed that the tumour mass filling the apical bronchus of the lower lobe was a well differentiated leioI myoma. The ovoid-shaped nuclei were uniform in shape and size and their chromatin was finely and regularly dispersed. The interlacing bundles formed by these tumour cells were fairly broad and did not change direction frequently or mingle int:mately with one another. Considerable importance attaches to this, and the growth pattern of this tumour was slightly more regular in this respect than were some of the other myomata of extrauterine origin seen in the pathological department which 
had metastasized widely. There were no abnormal nuclear forms and no multinucleated cells. There were only a few areas of necrosis. Multiple sections showed the tumour growing into the bronchial lumen and not extending beyond the connective tissue or cartilage of the bronchial wall. Bronchial epithelium was preserved over the surface of the tumour, except where this projected into the main lower lobe bronchus. Here the epithelium was deficient in areas and the surface presented granulation tissue which sometimes showed a low-grade inflammation. Often the tumour tissue was attached only over a small segment of the bronchial circumference, but grew into the lumen so that this was reduced to a narrow, cleftlike space lined by bronchial epithelium. Sometimes this bronchial epithelium over the surface of the tumour showed squamous metaplasia (Fig. 4).

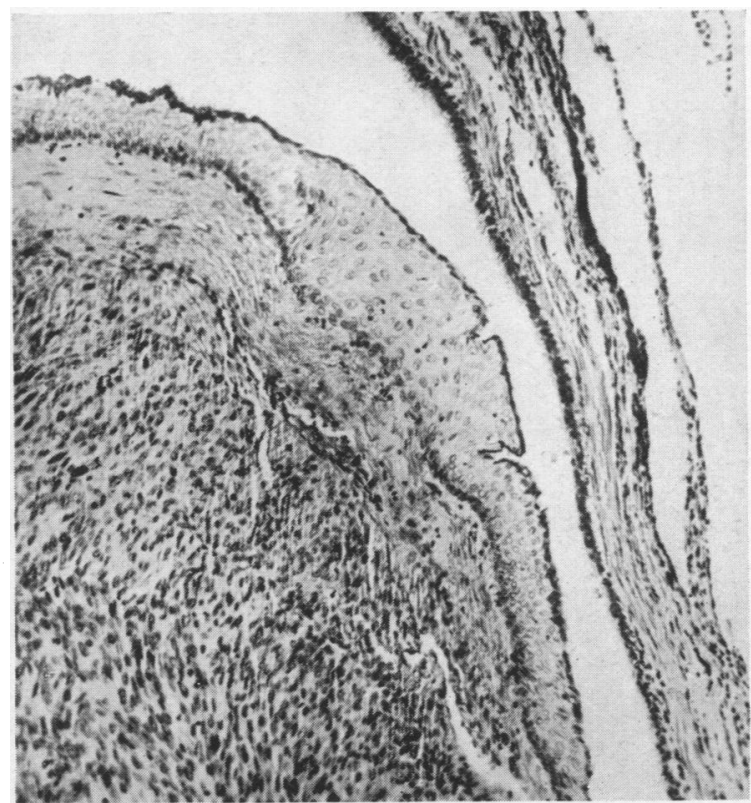

FIG. 4.-The epithelial covering of the tumour in this bronchus has undergone squamous metaplasia. The arrangement of the tumour cells in relatively wide interlacing bundles can be seen. Haematoxylin and eosin, $\times 90$.

The firm nodules noted grossly throughout the lung were small areas of organizing pneumonia of varying age, sometimes associated with small collections of lipoid-filled macrophages in the air spaces. There was no tumour tissue which was not directly continuous with the mass in the bronchus, and it is unlikely that the tumour was a single metastasis from a myoma elsewhere in the body. Sections of lymph nodes showed only a non-specific inflammatory change and a very moderate amount of carbon pigment.

\section{Discussion}

In view of the association of trauma with the onset of symptoms and the clinical findings of a massive pulmonary collapse in this case, it was essential to consider a possible fracture of the bronchus. Lesions of this type are most commonly produced by crush or compression injuries of the chest wall. Lloyd Griffith (1949) has pointed out that a severe shearing force can snap the relatively rigid bronchus, while the softer structures, such as the lung tissue proper and the vessels, may escape damage. Complications such as fractured ribs, pneumothorax, and haemothorax are not invariably associated with these fractures of the bronchi. On the other hand, as the main bronchus is always involved, pulmonary collapse with mediastinal displacement is a constant finding in the reported cases. The diagnosis depends on bronchoscopic examination. In our case fracture was excluded, and the presence of tumour cells in the biopsy specimen was significant. 
Leiomyoma is an innocent tumour of plain muscle. It is so common in the uterus, in the reproductive phase of life, that Boyd (1947) described it as the commonest tumour in the body; but it is uncommon in extrauterine sites where smooth muscle occurs. McFarland (1935) stated that myomata had yet to be described arising from the iris, ciliary body, bronchial tubes, pancreatic ducts, or spleen. A search of the present-day literature reveals that this is still true so far as the tracheo-bronchial tree is concerned.

Forkel (1910) described a fibro-myoma found incidentally at necropsy in the apex of the left lung of a woman aged 63 years who had died from pneumonia. The tumour was firmly adherent to the parietal pleura, to the vertebral bodies, and to their corresponding ribs. He considered that the mass had an intrapulmonary origin, because when separated there was no line of cleavage between the tumour and the lung, the exposed surface of the latter being composed of alveoli, bronchi, and blood vessels. He assumed that it arose from the normal smooth muscle of the lung, and considered especially the walls of the bronchi and the vessels. The possibility of a metastasizing tumour does not appear to have been considered.

A case of leiomyoma of the lung which occurred in association with pulmonary tuberculosis was reported by Brahdy (1941). A discrete shadow at a lung base was discovered on a routine radiograph. It was observed to move with respiration on fluoroscopic examination. As the sputum was positive, it was thought to be a tuberculoma. Lobectomy was performed, and the tumour was found to be a leiomyoma. Unfortunately, the exact pathological site of origin of this tumour was not stated. It is well known that secondary myomata occur in the lung and elsewhere, having metastasized via the blood stream from an extrapulmonary primary source as described by McFarland (1935). It is necessary to keep such a possibility in mind when a myoma is encountered in the lung. We regret that we were unable to obtain for review a thesis on multiple primary myomas of the lung by Deussing (1912).

Leiomyoma is not related to the so-called myoblastoma of Abrikossoff (1926). This tumour was named by him and claimed to arise from embryonal striated muscle cells or myoblasts. He laid down certain histological criteria, and tumours stated to conform to these have been described in a variety of sites by various observers. Kramer (1939) and Kraus, Melnick, and Weinberg (1948) recorded two such tumours arising in the bronchial tree, an ectopic site if the relationship to striated muscle is admitted. Grayzel and Friedman (1937) postulate a dysontogenetic origin for cells of the myoblastoma, implying that, during the early stages of development, primordial muscle cells of the myotomes become included within cell complexes that migrate to form other structures. The myogenous derivation of myoblastoma in such a site is questioned by Gray and Gruenfield (1937), who infer that the similarity of the histological pictures is the only reason for claiming relationship with striated muscle for this tumour. The leiomyoma is a more clear-cut histological entity.

\section{SUMMARY}

A case presenting with bronchial occlusion following trauma is described and a differential diagnosis is discussed, including fracture of the bronchus as a cause of pulmonary collapse. 
Pneumonectomy was performed, and pathological examination of the lung revealed a primary leiomyoma of bronchial origin.

Search of the available literature indicates the extreme rarity of such a tumour in this site. Brahdy (1941) and Forkel (1910) have described cases of leiomyoma and fibromyoma respectively, but in neither case is the exact pathological site of origin clear. The importance of considering an extrapulmonary site of origin for a primary tumour is stressed, and especially in women the necessity for a full pelvic examination will be readily understood.

Distinction is made between this tumour and myoblastoma related by some to striated muscle.

We wish to thank Mr. G. R. B. Purce, M.Ch., F.R.C.S., for his advice in the surgical management of this case ; J. Edgar Morison, B.Sc., M.D., for the pathological report and his advice; and Mr. D. McA. Mehaffey, A.R.P.S., who was responsible for the photography.

\section{REFERENCES}

Abrikossoff, A. (1926). Virchows Arch., 260, 215.

Boyd, W. (1947). Textbook of Pathology. London: Henry Kimpton. 5th Edition, P. 288.

Brahdy, L. (1941). Amer. Rev. Tuberc., 43, 429.

Duessing, R. (1912). "Multiple Primäre Myome der Lunge." Thesis, University of Munich. Forkel, W. (1910). Z. KrebsForsch., 8, 390.

Gray, S. H., and Gruenfeld, G. E. (1937). Amer. J. Cancer, 30, 699.

Grayzel, D. M., and Friedman, H. H. (1941). Arch. Pathol,, 31, 512.

Kramer, R. (1939). Ann. Otol., etc., St. Louis, 48, 1083.

Kraus, A. R., Melnick, P. J., and Weinberg, J. A. (1948). J. thorac. Surg., 17, 382.

Lloyd Griffith, J. (1949). Thorax, 4, 105.

McFarland, J. (1935). Amer. J. Cancer, 25, 530. 